\title{
Belphégor
}

Littérature populaire et culture médiatique

17 | 2019

Mutations des légitimités dans les productions

culturelles contemporaines

\section{Les savoirs des lecteurs de manga sur les sites non légaux : construction et diffusion de l'interprétation}

\section{Bounthavy Suvilay}

\section{(2) OpenEdition}

\section{Journals}

Electronic version

URL: https://journals.openedition.org/belphegor/1809

DOI: 10.4000/belphegor.1809

ISSN: 1499-7185

Publisher

LPCM

Electronic reference

Bounthavy Suvilay, "Les savoirs des lecteurs de manga sur les sites non légaux : construction et diffusion de l'interprétation", Belphégor [Online], 17 | 2019, Online since 18 April 2019, connection on 25 August 2021. URL: http://journals.openedition.org/belphegor/1809 ; DOI: https://doi.org/10.4000/ belphegor.1809

This text was automatically generated on 25 August 2021

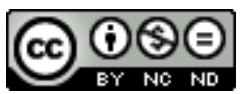

Belphégor est mis à disposition selon les termes de la Licence Creative Commons Attribution - Pas d'Utilisation Commerciale - Pas de Modification 4.0 International. 


\title{
Les savoirs des lecteurs de manga sur les sites non légaux : construction et diffusion de l'interprétation
}

\author{
Bounthavy Suvilay
}

1 En sociologie des médias et en études de la réception, de nombreuses recherches ont déjà été réalisées sur les écrits des amateurs dans les forums, les blogs ou les sites promouvant des critiques ${ }^{1}$. Cet intérêt est lié au fait que le terrain d'enquête choisi n'est ni induit ni construit par le chercheur qui se contente de délimiter son observation dans le temps ${ }^{2}$. Sans perturber les interactions par ses interventions ou sa simple présence, il peut alors collecter un corpus d'interprétations sociales d'œuvres consacrées ou appartenant à la culture populaire. Henry Jenkins a étudié les réactions de fans de Twin Peaks sur le groupe de discussion alt.tv.twinpeaks ${ }^{3}$, Matt Hills a analysé celles des téléspectateurs de X-Files sur alt.tv.X-Files ${ }^{4}$, Laurence Allard s'est intéressée à celles du groupe Fr.rec.cinema.discussion à propos de Titanic ${ }^{5}$, Beaudoin et Pasquier ont analysé les critiques du site qu'elles choisissent d'appeler «Viv@ films ${ }^{6}$. Ces recherches mettent en avant la présence de deux formes principales de critique : l'une est centrée sur l'expérience émotionnelle, l'autre, plus érudite, se rapproche de la critique professionnelle tant au niveau de la formulation que des critères d'évaluation. Comme pour d'autres études sur les pratiques sur internet, ces recherches soulignent que la majorité des contributions sont réalisées par un nombre restreint d'utilisateurs, ce qui remet un peu en question l'image d'une création démocratique du savoir.

2 L'interface d'un site programmant un modèle de sociabilité, nous nous sommes intéressés à Disqus (interface conversationnelle publique) et au site non légal Kissmanga $^{7}$. Au lieu d'analyser les processus de production et de validation du savoir sur des sites participatifs ${ }^{8}$, il nous semblait intéressant de montrer comment la conversation médiatisée par un dispositif socionumérique pouvait produire des savoirs qui sont ensuite relayés sur d'autres plateformes en ligne. Il ne s'agit plus de la 
conversation de visu au sujet d'un objet culturel telle que Dominique Boullier a pu l'étudier à propos de la télévision ${ }^{9}$ ' mais des réactions en ligne immédiatement après lecture d'un chapitre, ce que Hills nommait le «just-in-time fandom $»^{10}$. D'autre part, ces conversations se forment dans un espace non territorialisé où des personnes de multiples pays peuvent échanger leurs points de vue sans s'être jamais rencontrées. Dans leur étude de la réception de Dallas, Liebes et Katz avaient souligné que si la série est bien diffusée à travers le monde, elle prend des significations différentes pour les téléspectateurs selon qu'ils habitent au Japon ou à Israël ${ }^{11}$. Ceux-ci s'appuient sur différents systèmes de croyances et références culturelles pour construire le sens de cette fiction américaine. Nous nous demanderons dans quelle mesure le système de communication transnational qu'est Disqus, implémenté sur le site non légal Kissmanga, offre la possibilité de construire de nouvelles formes de signification d'une œuvre japonaise au sein d'une communauté d'interprétation anglophone. Quels sont les types de savoirs produits lors de ces conversations asynchrones en ligne? Après avoir présenté la plateforme de lecture et l'outil de commentaires, nous tenterons de catégoriser les différentes formes de savoirs construits par la communauté d'utilisateurs et en quoi celles-ci sont liées au plaisir de la lecture et de son partage en ligne.

\section{Présentation de la plateforme}

3 Il nous semblait pertinent pour une analyse de la réception de s'intéresser au dispositif technique et au type de savoir généré par les discours d'amateurs. La spécificité de notre terrain porte sur deux points. Contrairement au forum ou au site spécialisé détaché du lieu de consommation (type Reddit), Kissmanga est une plateforme de lecture en ligne non légale proposant sur une même page le chapitre de manga traduit en anglais par des fans et les commentaires des internautes lecteurs ${ }^{12}$. Il propose à la fois un dispositif de dialogue synchrone par le biais d'une messagerie instantanée (utilisant le logiciel Discord) et un second asynchrone par le biais de la plateforme Disqus, que l'on peut rapidement décrire comme étant un Facebook des commentateurs, la mise en relation ne se faisant pas en fonction des personnes appartenant réellement à son entourage mais en fonction de l'homophilie se manifestant à travers les commentaires ${ }^{13}$. Dans le cadre de cette étude, nous nous intéresserons seulement aux commentaires des lecteurs par l'interface Disqus qui ajoutent des fonctionnalités propres aux réseaux socionumériques, comme les marques d'appréciation ou de recommandation exprimées à travers le bouton « +1 » et les outils de sélection des messages, de mise en relation avec un autre lecteur, etc. 
i literally live for these moments where hinata and kageyame go god-mode and surprise the shit out of their opponents/spectators

AND LOOK HINATA'S FINALLY GETTING TO THE POINT WHERE HE AND KAGEYAMA MAKE SIMILAR GAME DEDUCTIONS THEY'RE GONNA BE SO IN SYNC BY THE TIME THEY'RE 3RD YEARS... THE MOST TERRIFYING DUO ON THE CIRCUIT

$183 \wedge \vee$. Reply . Share

kukuku $\nrightarrow$ ShiningRegalia $\cdot 7$ months ago

lol they are not in sync, they are literally competing each other, kageyama didnt want to lose to hinata and the same goes for hinata, it make them look like in sync Imao

$40 \wedge \checkmark$. Reply. Share

SAYURI $\rightarrow$ kukuku $\cdot 7$ months ago

i can't agree more... i mean who even compete for the fastest to clean up the floor. Only them.

$17 \wedge \vee$. Reply $\cdot$ Share

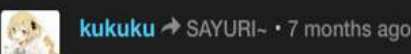

totally true lol

FIgURE 1 : SUR LES 135 COMMENTAIRES dU CHAPITRE 250 dE HAIKYÛ!!, CELUI-CI A REÇU 183 « +1 » ET PLUSIEURS RÉPONSES QUI S'ENCHAÎNENT.

L'intérêt de Disqus est qu'il est un réseau social promouvant les conversations et non les textes critiques. Il privilégie les formats d'énonciation brefs rapprochant l'écriture en ligne des formes oralisées de la conversation ordinaire. Il s'agit d'une chambre numérique où le lecteur peut échanger avec d'autres ses idées, d'un espace public où s'établissent des dialogues délibératifs dont les contenus peuvent ensuite être repris et développés sur d'autres réseaux sociaux et d'autres plateformes de diffusion de contenus : blog, vidéo youtube, wiki ${ }^{14}$. Il est difficile de suivre les conversations des lecteurs d'une série sur les réseaux sociaux à moins que celles-ci soient publiques et signalées par un hashtag ${ }^{15}$. Par le biais de Disqus, nous avons en quelque sorte un équivalent de ces conversations, à ceci près qu'elles se déroulent entre des personnes partageant la lecture d'une même œuvre et qu'elles suivent presque immédiatement la pratique de la lecture ${ }^{16}$.

5 Le deuxième élément particulier de l'étude est qu'elle porte sur des œuvres japonaises, traduites et lues par un public anglophone (que l'anglais soit leur langue maternelle ou une langue seconde). Rappelons que le public de ces plateformes parallèles est difficile à définir en raison de l'anonymat lié aux pseudonymes et de la faible pérennité des sites migrant régulièrement d'une adresse à une autre. Néanmoins, à travers les commentaires laissés il est possible de reconstituer des profils d'utilisateurs. Sur cette plateforme anglophone possédant une URL russe, on trouve notamment les commentaires d'un amateur de volleyball brésilien regardant une série japonaise (Haikyû!! $\left.{ }^{17}\right)$, ce qui est la preuve de l'importance de l'aire linguistique par rapport à l'appartenance à un territoire physique ${ }^{18}$. 
Thomaz Barros Costa $\cdot 8$ months ago

It's funny hearing that story about the Ceiling Serve.

Here in my country we've been told that that play was innovated by one Bernard Rajzman in the 80 s.

Thanks to the height here it was nicknamed the "Jornada nas Estrelas", the brazilian name for "Star Trek".

It's a very iconic thing in the history of our volleyball.

Not that $\mathrm{i}$ know a whole lot about volley tho Imao

$2 \wedge \vee \cdot$ Reply . Share

Q. grazim $\rightarrow$ Thomaz Barros Costa $\cdot 8$ months ago

15. Yeah i was about to google that myself, not sure about how accurate the author was being there... Reply - Share

Figure 2 : Discussion à propos du « ceiling serve » du chapitre 240 et sur la réalité des techniques mises en scène dans le manga. L'utilisateur se présente comme Brésilien et la conversation se fait en anglais avec d'autres personnes dans un espace déterritorialisé où la langue véhiculaire est plus importante que le pays d'origine.

Les mangas disponibles ne sont pas tous traduits sur les territoires des lecteurs et il n'existe pas vraiment de presse spécialisée pour les promouvoir, donc pas vraiment de modèle de rédaction pour une critique. Il s'agira ici d'analyser plus précisément quel type de savoir et de valeur les amateurs convoquent lorsqu'ils sont face à une œuvre n'ayant pas de distribution officielle et donc pas de (contre-)modèle critique officiel pour (in)valider leur interprétation. Que se passe-t-il quand des inconnus interagissent au sein d'une communauté polyglotte en commentant une œuvre transculturelle traduite et diffusée par des amateurs?

7 Pour le site Kissmanga, le dispositif de commentaires sert avant tout à retenir et fidéliser les utilisateurs, l'accroissement de leur nombre augmentant la valeur des pages de publicité qu'il peut proposer aux annonceurs. L'interface du site programme un modèle de sociabilité, celui de la conversation spontanée faisant suite à la lecture ${ }^{19}$. Pour les lecteurs, le dispositif permet d'engager des conversations avec une communauté homophile. En se rendant chaque semaine sur le site pour lire le nouveau chapitre de manga, ils constituent un véritable public dans un espace numérique asynchrone qui évoque les " communautés imaginées ${ }^{20}$ de Benedict Anderson. Si dans le monde analogique les lecteurs de journaux du XIX siècle pouvaient former un public, partager une même actualité, et se constituer en nation, dans le monde numérique, les individus partagent les mêmes goûts et enthousiasmes dans un lieu déterritorialisé.

\section{Savoir émotionnel}

8 Si l'on se fie au dispositif technique (nombre de +1 ), les discours les plus appréciés sont essentiellement des commentaires de type émotionnels, le public réagissant aux événements de la diégèse ou à l'interruption liée à un cliffhanger. L'appréciation de l'utilisateur ShiningRegalia sur la synchronisation du jeu des deux héros au chapitre 250 de Haikyû!! a par exemple recueillis 180 voix et 12 réponses (fig. 1) ${ }^{21}$. Les lecteurs décrivent essentiellement leur ressenti, dressent la liste des moments qu'ils ont le plus appréciés, les manifestations de leur plaisir face au visionnage (fig. 3). Outre les exclamations et jargon liés au dialogue en ligne, beaucoup décrivent leur réaction physique face à la fiction (fig. 4). Les contributions ont ainsi pour principale motivation le désir de partager les émotions suscitées par la lecture. Les institutions scolaires 
françaises essaient précisément de renouer avec ce plaisir à travers les débats interprétatifs et la notion de sujet-lecteur ${ }^{22}$. Mais dans le cas des commentaires en ligne, ce plaisir se formule à travers une ponctuation excessive, des formulations outrancières, des éléments graphiques (emoticon, kaomoji, gif animé) comme pour pallier l'absence physique et le manque d'interconnaissance.

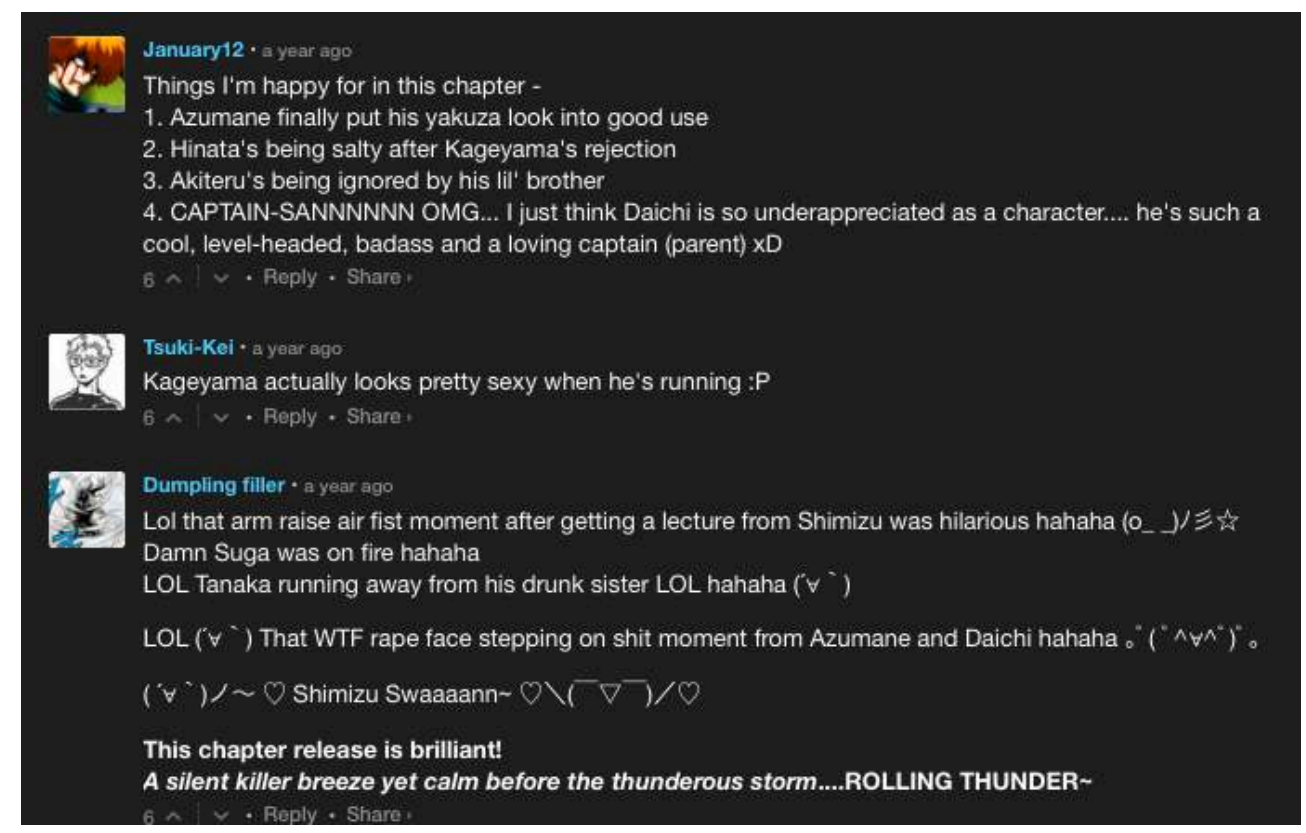

Figure 3 : Deux formes d'énumération des moments les plus appréciés dans la lecture du chapitre 229 : la version sobre de January 12 (liste numérotée) et celle plus expressive de Dumpling filler (abréviation, kaomoji et texte en gras).

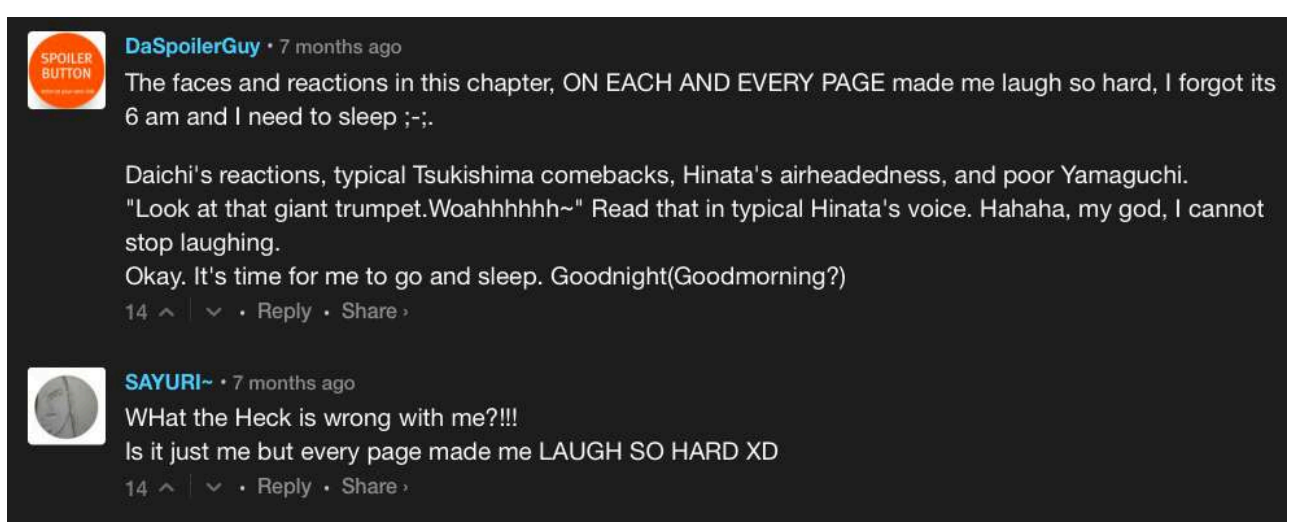

Figure 4 : L'utilisateur récapitule les éléments qu'il a le plus appréciés et les réactions physiques provoquées par la lecture (rire et absence de sommeil) comme pour pallier le manque d'interaction physique sur cette plateforme numérique.

9 De plus, en lisant ces réactions validées par d'autres internautes, le lecteur est incité à partager les mêmes comportements. Dans leur théorie de la communication à double étage, Katz et Lazarsfeld estimaient que les médias n'influençaient pas directement les publics mais que l'information diffusée était relayée par des leaders d'opinion bénéficiant d'une forte influence interpersonnelle ${ }^{23}$. Dans Disqus, ces leaders ne sont pas des personnes que les lecteurs connaissent personnellement, mais des individus possédant une bonne réputation au sein de la communauté de lecteurs. La mise en valeur des «meilleurs» commentaires par le dispositif technique favorise ainsi l'influence de certains utilisateurs qui instituent, sans nécessairement le vouloir, une 
forme de recommandation quant au bien agir face à la fiction, aux formes correctes d'appréhender subjectivement le récit : être angoissé ou heureux, pleurer de joie ou de tristesse. Ces commentaires viennent valider les dispositifs textuels au sein de l'œuvre visant à créer une tension narrative, à provoquer le suspens ou la curiosité. Ces descriptions d'émotions constituent un savoir bien lire, préalable indispensable au savoir analyser.

10 Il serait facile de voir dans ces réactions une satisfaction primaire, loin du plaisir supposé plus subtil des œuvres légitimes ou du lecteur savant. En réalité, le fait de décrire une réaction émotionnelle identique à celle des autres récepteurs sert à renforcer l'effet de cohésion du groupe en tant que public. L'affichage des goûts et dégoûts (pour un personnage, une action) est un moyen de se constituer en public. Citant Hannah Arendt, Allard estimait que ce type d'utilisateurs en ligne constituent des « compagnies » : « En communiquant ses sentiments, on révèle ses choix, on élit sa compagnie $»^{24}$. Le partage de la lecture fait ainsi partie du plaisir de cette pratique en ligne $^{25}$. En ce sens, les commentaires en ligne ne sont que le prolongement de ce qui se faisait déjà dans le monde analogique où les gens regardent moins la télévision pour son contenu que pour les conversations auxquelles ils peuvent ensuite participer dans leur vie sociale ${ }^{26}$. L'affichage de l'affect constitue ces lecteurs en un public s'exprimant de concert dans une sorte d'intimité communautaire.

11 C'est seulement après avoir montré aux autres que l'on appartient à la même communauté de goût qu'il est possible d'avoir un dialogue délibératif, plus abstrait. La reconnaissance entre personnes de même goût précède et instaure la possibilité d'un espace public de discussion. Le partage de l'émotion et le fait de formaliser aux regards des autres cette émotion commune constitue le public en tant que communauté interprétative consciente d'elle-même. Gabriel Tarde nommait « sensation d'actualité » le phénomène liant les lecteurs de la presse quotidienne, la création d'une sociabilité à distance avec des personnes partageant un même intérêt pour une question d'actualité ${ }^{27}$. Benedict Anderson mentionnait l'importance des journaux et des romans dans la création d'une "communauté imaginée» au niveau du lectorat de ces imprimés. Dans le cas des mangas, feuilletons élaborés pour que chaque chapitre s'achève sur un cliffhanger, la sensation d'actualité et la lecture synchrone d'un même journal est remplacée par la joie asynchrone suscitée par le récit séquentiel consulté selon les modalités choisies par l'individu, de l'enthousiasme partagé face aux événements de la diégèse et la manière de les présenter. Les commentaires émotifs témoignent de l'invention d'un mode de subjectivation collective dans la pratique commune du plaisir de la fiction, d'une connexion affective qui précède la rationalisation.

\section{Savoir diégétique}

12 Une fois le plaisir partagé de la lecture affirmé, les lecteurs discutent au sujet du monde diégétique. Leurs commentaires tendent à construire une cohérence là où le manga se montre plus évasif: ils comblent les blancs laissés par le texte et par le biais de comparaisons ou de demandes d'information successives construisent une encyclopédie non formalisée.

13 De nombreux lecteurs poursuivent l'énonciation de leur goût pour tel ou tel personnage en donnant une interprétation psychologique de ses actions, en comparant 
leur talent avec d'autres figures du manga. L'utilisatrice Kaia explique ainsi que les personnages Hinata et Tsuki ne se détestent pas, qu'ils s'entendent même mieux qu'au début du manga (fig. 5). Elle appuie son propos en faisant référence aux chapitres et à la fonction des deux joueurs au sein de l'équipe. Le raisonnement est mis en œuvre pour justifier les propos après la mise sous observation subjective de la fiction. Les différents lecteurs déduisent les relations entre personnages à partir des dialogues et des commentaires des autres lecteurs. Ces nombreux discours sur le personnage contribuent à lui donner une densité qu'il n'avait peut-être pas ${ }^{28}$, tout en participant à l'élaboration d'un système de valeurs pour les membres de ce public particulier, qui peuvent ensuite se positionner en son sein.

Discussion on KissManga · 105 comments

\section{Read manga Haikyuu!! Chapter 258 online in high quality}

Kaia $\rightarrow$ Eireia Lavosia * a year ago

To be honest, I thought he was going to say Kuroo since he's so well known for his blocking, lol! But as players, I don't think Hinata hates Tsukki, it's more like a rivalry due to playing the same spot and a stark contrast in their personalities that tend to clash. They've begun to get along marginally better though, as we saw a chapter or two ago when Hinata got that block and asked if Tsukki was jealous only to be met with a sarcastic kind of "you got me there" reply. Also, Tsukishima's kind of blocking isn't something Hinata will be able to successfully emulate, so that ruled him out for me while trying to figure out who he could've meant. For one, Hinata isn't as calculating and observant (not as he is now anyways lol)

$1 \uparrow \downarrow$ Reply View in discussion

Figure 5 : Interprétation psychologisante des personnages basée sur leurs comportements dans de précédents chapitres.

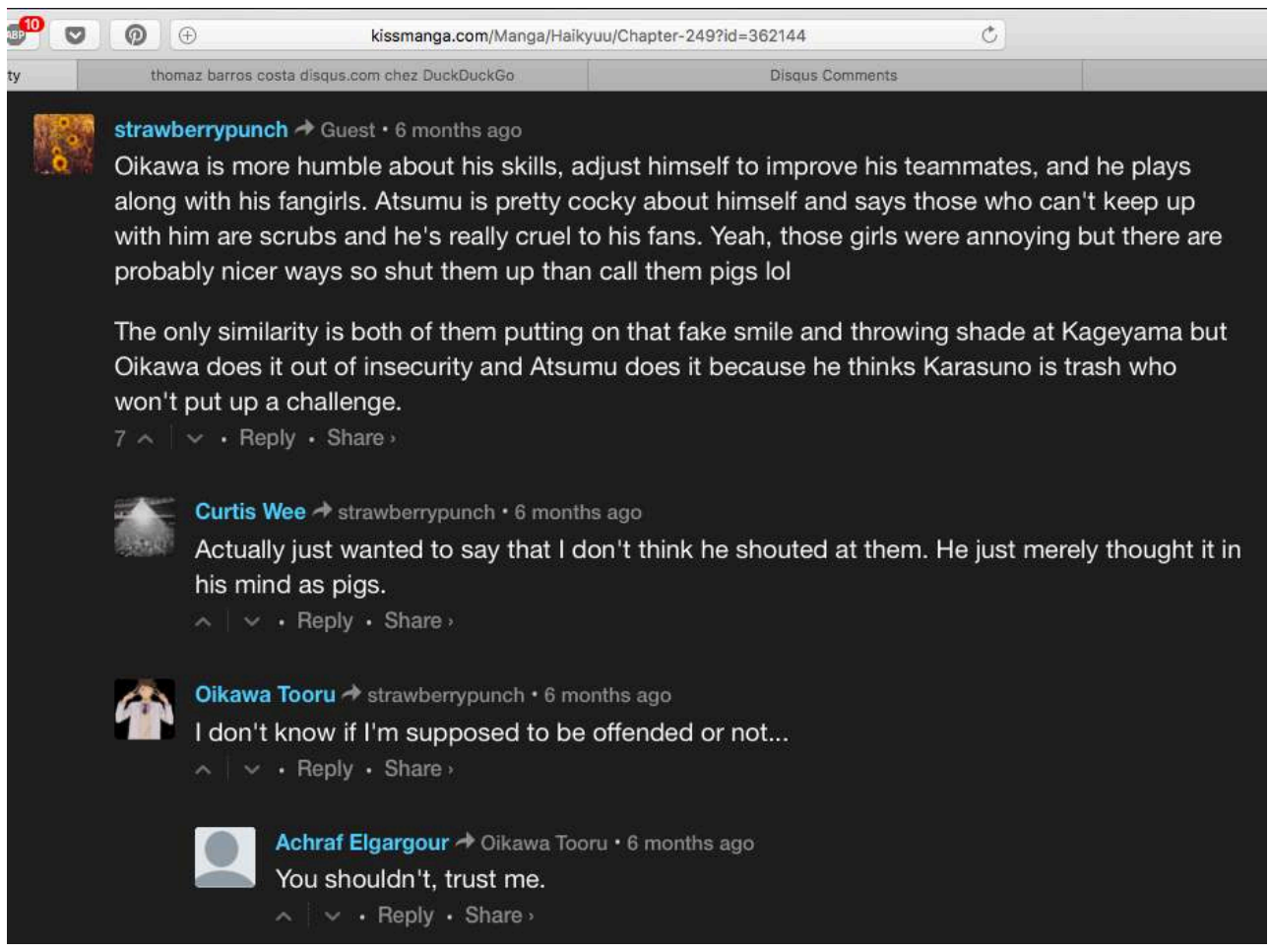

Figure 6 : Au chapitre 249, des lecteurs comparent les attitudes de deux personnages (Oikawa et Atsumu) en donnant des justifications psychologiques. Le dialogue est suivi par l'intervention de l'un deux personnages (Oikawa, incarné par un utilisateur en Role Play). 

différents personnages, informations qui peuvent ensuite être sédimentées dans des wikis ou d'autres sites de fans qui constituent une version archivée des informations issues des conversations. Ainsi, sur le wiki anglophone de la série, la description du personnage d'Oikawa est extrêmement détaillée et comporte un onglet pour les relations qu'il entretient avec les principaux personnages de la série ${ }^{29}$. Ce que Richard Saint Gelais nomme la "pulsion archivistique des fans " $^{30}$ s'exprime bien avant la mise en place d'encyclopédie et de définition du canon : elle est liée au plaisir de la lecture et à la justification de ses goûts au sein d'un public restreint d'homophiles. De fait, le canon s'établit à travers la confrontation des goûts dans le cercle conversationnel des amateurs.

15 A cette densification du personnage par le biais du rappel de ses actions et dialogues afin de créer une cohérence psychologique (qu'il n'avait pas toujours) s'ajoute une pratique plus ludique où le lecteur prend le nom du personnage comme pseudonyme, comme avatar ou comme incarnation dans une forme de jeu de rôle (Role Play an anglais) (fig. 6). Face aux paroles d'encouragement d'un lecteur adressé aux personnages, deux utilisateurs ayant pour pseudonyme Nishinoya lui répondent comme s'ils étaient le joueur sorti du manga pour lui parler (fig. 7).

msycn $\cdot 7$ days ago

Ennoshita!! Calmgeyama - as usual Hinata's all about Tobio. Tsukki, show some bad-assery please. Nishinoya we are counting on you. Asahi's being cool.

$14 \wedge \vee$. Reply . Share

6. Nishinoya Yuu $\rightarrow$ msyon $\cdot 7$ days ago

(1) YOU CAN COUNT ON ME!!

(if you just call me Nishinoya senpai XD)

I'VE GOT THEIR BACKS AFTER ALL!!!

$2 \wedge \checkmark$. Reply. Share

(N) Roon Roollinnngg Thunderrrrr!!! $\rightarrow$ msycn $\cdot 7$ days ago

Yes! Yes! Let karasuno's deity handle it from the back! He's got this!

$1 \wedge \vee$. Reply . Share

Figure 7 : Au chapitre 275 les utilisateurs « Nishinoya Yuu » et « Roooollinnngg Thunderrrrr !!! » (deux incarnations ludiques du même personnage) répondent à l'utilisateur « msycn ». Nishinoya Yuu est le nom du personnage et « Roooollinnngg Thunderrrrr » est l'une de ses phrases récurrentes.

16 D'autres personnages sont ainsi interprétés par des lecteurs qui peuvent produire des dialogues similaires à ceux qui existent dans le manga ${ }^{31}$. Cette activité ludique contribue à accroître la convivialité de l'espace de discussion, à souder les lecteurs autour des mêmes références. Cette écriture inventive témoigne de la bonne maitrise des données sur le personnel de fiction. Il ne s'agit pas encore de fan fiction à proprement parler mais de cas d'appropriation créative des éléments de la diégèse par le biais d'un jeu de rôle. D'autres utilisateurs emploient leur savoir sur la fiction pour créer des jeux de mots dans les dialogues entre lecteurs. Nous avons également trouvé un poème pour célébrer un chapitre. Ces multiples formes de jeu permettent d'accroître la connivence entre les membres de la communauté, de confirmer la connaissance personnelle de l'univers diégétique, et de s'imposer comme une sorte d'auteur capable de prendre en charge la poursuite de la fiction.

$\mathrm{Au}$ savoir spécifique sur les personnages, s'ajoutent des connaissances sur le monde diégétique: sa proximité avec le monde réel, sa cohérence dans le manga et dans l'ensemble des récits disponibles sur d'autres supports. Dans le cas de Haikyû!!, outre la 
construction détaillée du réseau de relations entre les joueurs des différentes équipes, la vraisemblance des actions sportives décrites est régulièrement questionnée dans les commentaires. Il s'agit de recenser les écarts entre le monde diégétique et le monde réel. Les erreurs de compréhension factuelle de certains lecteurs sont corrigées au fil de la discussion en faisant appel aux connaissances personnelles des utilisateurs, que ce soit à propos du système scolaire japonais, des règles du volley-ball, des dimensions des stades, des techniques... Au chapitre 239 et 240 , l'utilisation du " ceiling serve » par un joueur est commentée par des lecteurs incrédules et sa vraisemblance confirmée par d'autres internautes qui pratiquent ce sport (fig. 2). Le tout est par ailleurs commenté par d'autres lecteurs sur Youtube $^{32}$, ou divers forums ${ }^{33}$, avant d'avoir une entrée spécifique dans le wiki $^{34}$. Les différents commentateurs puisent dans leurs connaissances personnelles pour produire une argumentation et un savoir appliqué à la fiction. Bien sûr, même s'il leur arrive de faire des lectures référentielles, les commentateurs ne confondent en aucun cas les personnages avec des personnes réelles. Le plaisir de la lecture est justement de jouer avec la frontière entre fait et fiction, souligner les proximités ou les divergences pour savourer ce qui est plaisant au sein du récit.

18 Si la plupart des lecteurs de Haikyû!! sont plutôt admiratifs du travail de l'auteur, Haruichi Furudate, ce n'est pas le cas de ceux de Dragon Ball Super. Dans les conversations en ligne sur le site, les internautes critiquent régulièrement l'auteur pour ses incohérences scénaristiques. Un autre l'insulte car Goku a tué un adversaire, geste qu'il n'avait jusqu'alors jamais réalisé. Ce type de discussion peut ensuite se déplacer sur d'autres plateformes comme Youtube où plusieurs séries de vidéos argumentatives se répondent et tentent de justifier l'intégration ou non de tel personnage dans le récit "canon», ou de déterminer l'échelle de puissance des personnages.

19 À travers les dialogues les intervenants construisent un savoir spécifique sur l'univers diégétique et se portent garants de sa cohérence générique, graphique ou morale, et ce même contre l'auteur. C'est au nom de cette élaboration participative d'un canon que le créateur original peut être vivement critiqué selon sa conformité ou non à l'interprétation forgée par les lecteurs, à l'ordonnancement diégétique qu'ils ont construit à partir des données de l'œuvre originale et des éléments retenus dans les multiples variations transmédiatiques de la licence. Les conversations permettent ainsi d'établir un "canon », ensemble d'éléments appréciés et perçus comme cohérents par les amateurs qui s'en servent ensuite d'une part pour évaluer les différentes déclinaisons de l'univers de fiction et d'autre part pour critiquer l'auteur s'il s'éloigne de la construction élaborée par les fans.

20 La fonction-auteur n'a donc pas disparu dans les productions médiatiques modernes et la consommation non légale ne restreint pas son poids imaginaire. Mais l'autorité de l'auteur est contestée par les commentateurs amateurs au nom d'une plus grande valeur esthétique s'opposant aux réalités économiques de la production qui empêcheraient l'auteur de "s'exprimer librement». Il faut dire qu'au cours des conversations, les utilisateurs déploient un grand nombre de connaissances sur le processus de création du manga, les contraintes de production et le personnel créatif. Les commentaires permettent ainsi de diffuser un troisième type de savoir au sein de la communauté : un savoir sur le monde de la création. 


\section{Savoir sur les processus de création}

21 Outre le plaisir affiché de la fiction et la prescription d'une manière d'apprécier le récit, les commentaires regorgent d'informations témoignant des compétences génériques et des connaissances techniques des lecteurs de cette plateforme. Par exemple, suite à un chapitre consacré au développement d'un personnage secondaire dans Haikyû!!, les uns expliquent leur frustration de ne pas avoir la suite directe du championnat, tandis que les autres répliquent en soulignant le caractère récurrent du procédé et en démontrant l'intérêt de la séquence narrative pour accentuer la tension dramatique (fig. 8). Ils identifient le chapitre comme un élément nécessaire à la progression du manga, comme un intermède mineur ayant pour fonction de relâcher la tension avant le match suivant. Les commentateurs montrent une forme d'expertise dans la reconnaissance de mécanismes sériels, dans l'appréciation de la complexité des constructions narratives.

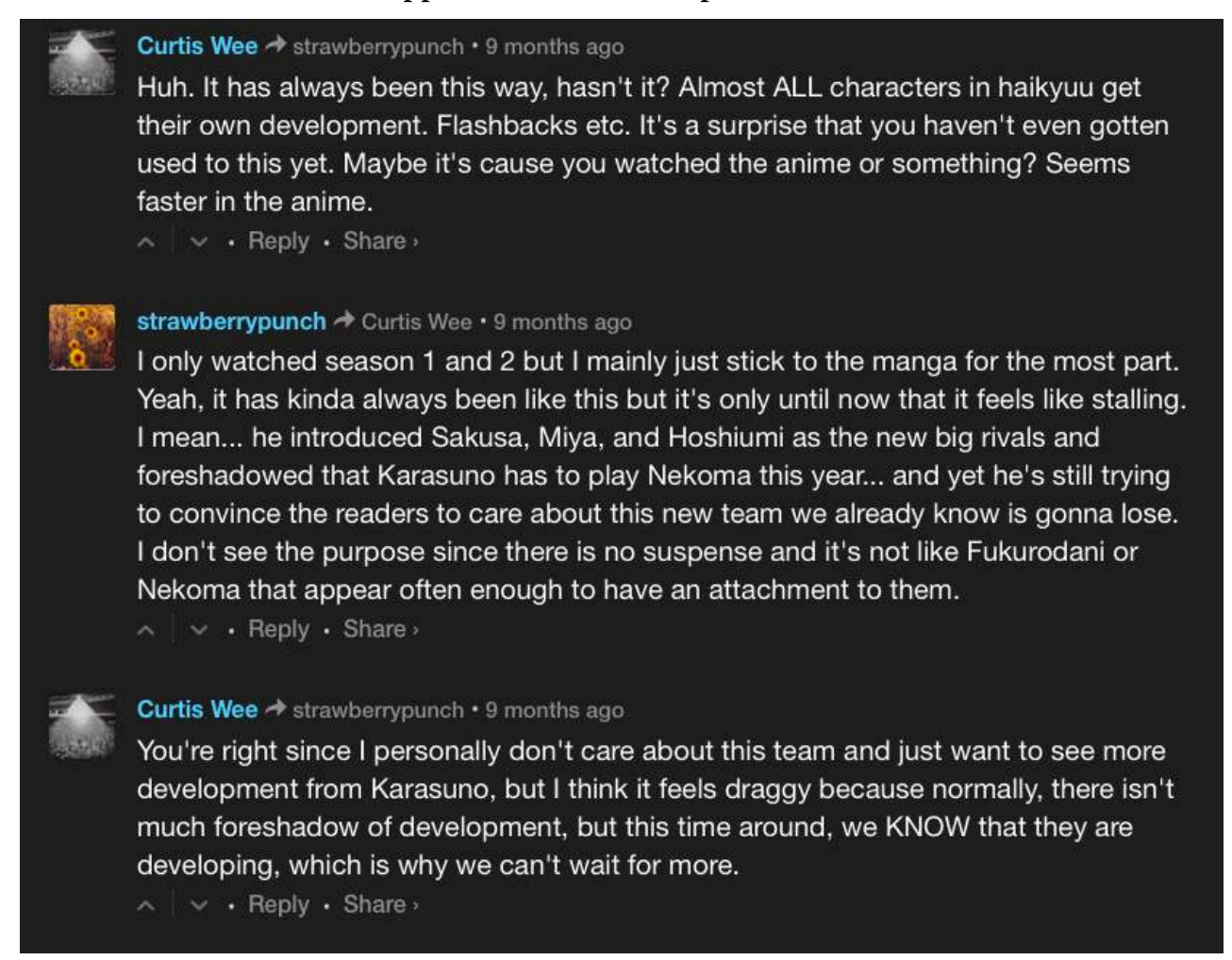

Figure 8 : Le chapitre 239 est perçu comme un développement inutile de la biographie d'un personnage secondaire. Curtis Wee souligne que c'est une technique récurrente à laquelle il est habitué mais que, dans ce cas précis, l'impatience est plus grande.

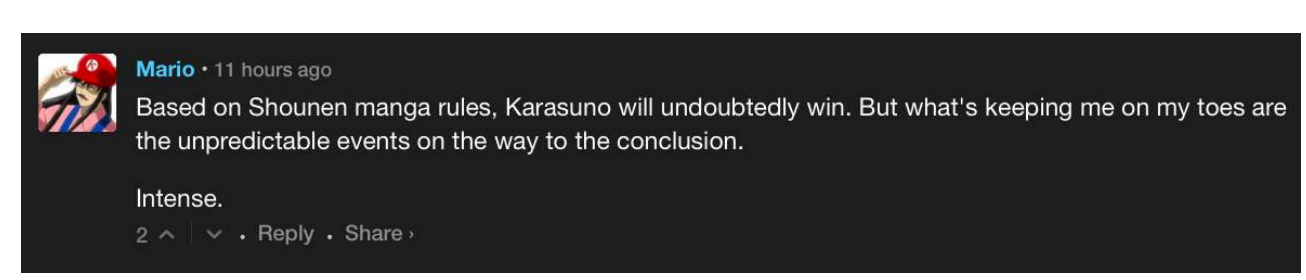

Figure 9 : Au chapitre 275, cet utilisateur précise qu'il connait la fin habituelle des mangas de sport mais qu'il continue de lire pour découvrir l'enchaînement des événements menant à la victoire. 
rikugou $\cdot 9$ days ago

Yeah, last time we had "just a captain" subbing for a normal player, we met Oikawa Töru. So no offense, but I'm not buying this "just a substitute" image.

$35 \wedge \vee$. Reply . Share

Nikhil Sharma $\rightarrow$ rikugou $\cdot 9$ days ago

But hey, karasuno was able to pull up against that mosnter + Captain + ace

Though they required two matches for the same

$2 \wedge \sim$. Reply - Share

rikugou $\rightarrow$ Nikhil Sharma $\cdot 9$ days ago

I'm not saying they'll lose (it's pretty obvious they'll end up playing in the finals or semi-finals with either Nekoma or Fukurodani). It's just that there are no "nothing to be afraid of" captains in sports mangas. The guy is here to make Karasuno's lives miserable in one way or the other.

$5 \wedge \mid \checkmark \cdot$ Reply $\cdot$ Share

Figure 10 : L'utilisateur souligne le fait que l'auteur a déjà utilisé la même technique auparavant et qu'il s'agit d'un cliché dans les mangas de sport : présenter le capitaine adverse comme un "simple joueur" (chap. 273)

Beaucoup font des pronostics sur les événements des chapitres à venir (fig. 10). Ceux-ci sont argumentés et font appel aussi bien à ce que certains nomment les "règles " du shônen manga (bien qu'en réalité elles n'existent pas en tant que codes établis) qu'à l'analyse des tomes précédents afin de souligner les habitudes de l'auteur (fig. 8 et 9). Certains commentaires comparent la série à d'autres mangas de sport afin de montrer en quoi elle s'en distingue (fig. 11). Ces comparaisons intertextuelles démontrent une connaissance permettant à la fois de définir le périmètre d'un genre et d'apprécier les écarts afin de montrer l'originalité du manga.

guiltycrown1 $\rightarrow$ Wairus $\cdot 21$ days ago

i have a hard time seeing them lose this tournament. Unlike other sports manga's this doesn't feel like a generational team. I mean for example Ace of Diamond is a show that has flowed several different generations of teams already. This manga hasn't really focused on bringing new team members in. I have not doubt this tournament is going to be heart wrenching and difficult, but $i$ cant not see them in any other scenario but winning it all. In my mind it would be very disheartening and cheap, especially to the third years, given how much time they are given.

$5 \wedge \sim \cdot$ Reply · Share

Axio $\rightarrow$ guiltycrown $1 \cdot 21$ days ago

Yeah, Haikyuu!! is more like Eyeshield21, that Ace of Diamond, I hope, they'll made it to a finals, like my lovely "Devil Bats". ^^^

But, at the other side, it's Haikyuu. Everything may happen here. :)

$2 \wedge \mid \vee \cdot$ Reply . Share

guiltycrown $1 \nrightarrow$ guiltycrown $1 \cdot 20$ days agc

also if you remember during the Shiratorizawa, Wakatoshi Ushijima indicates that hinata's style is different from the new little giant

A $\checkmark$. Reply Share :

Figure 11 : Spéculation sur la fin du match et comparaison avec d'autres mangas de sport (Eyeshield 21 et Ace of Diamond) (chap. 232)

23 Autrement dit, les récepteurs ne sont pas tous passifs ou facilement manipulables par les processus sériels, ils les comprennent parfaitement et certains parviennent à justifier leurs emplois ou à les critiquer auprès des autres membres du public. Par leur discussion, ils construisent un savoir générique sur le shônen manga et les séries de sports, préparant l'horizon d'attente d'autres lecteurs, alimentant les critiques sur d'autres supports. 
24 En dehors des commentaires critiques montrant une bonne connaissance des mécanismes du feuilleton, certains lecteurs font référence au cadre de publication pour souligner des détails dans un chapitre ou pour décrier la démarche commerciale du magazine de publication. Le chapeau de paille que porte l'un des personnages est explicité comme un hommage à One Piece (autre manga du magazine) à l'occasion de l'anniversaire de cette série. La durée des matchs s'étalant sur plus d'une vingtaine de chapitres est expliquée par le fait que Haikyû!! est l'une des séries phares et qu'il faut la prolonger (fig. 12).

\begin{tabular}{llll}
\hline d manga Haikyuu!l 239 - Ally online in high quality & kissmanga.com/Manga/Haikyuu/239---Ally?id=344592 & (1) Halkyul! Chapter 239 Review - The Celing Serve - \\
7 résultats
\end{tabular}

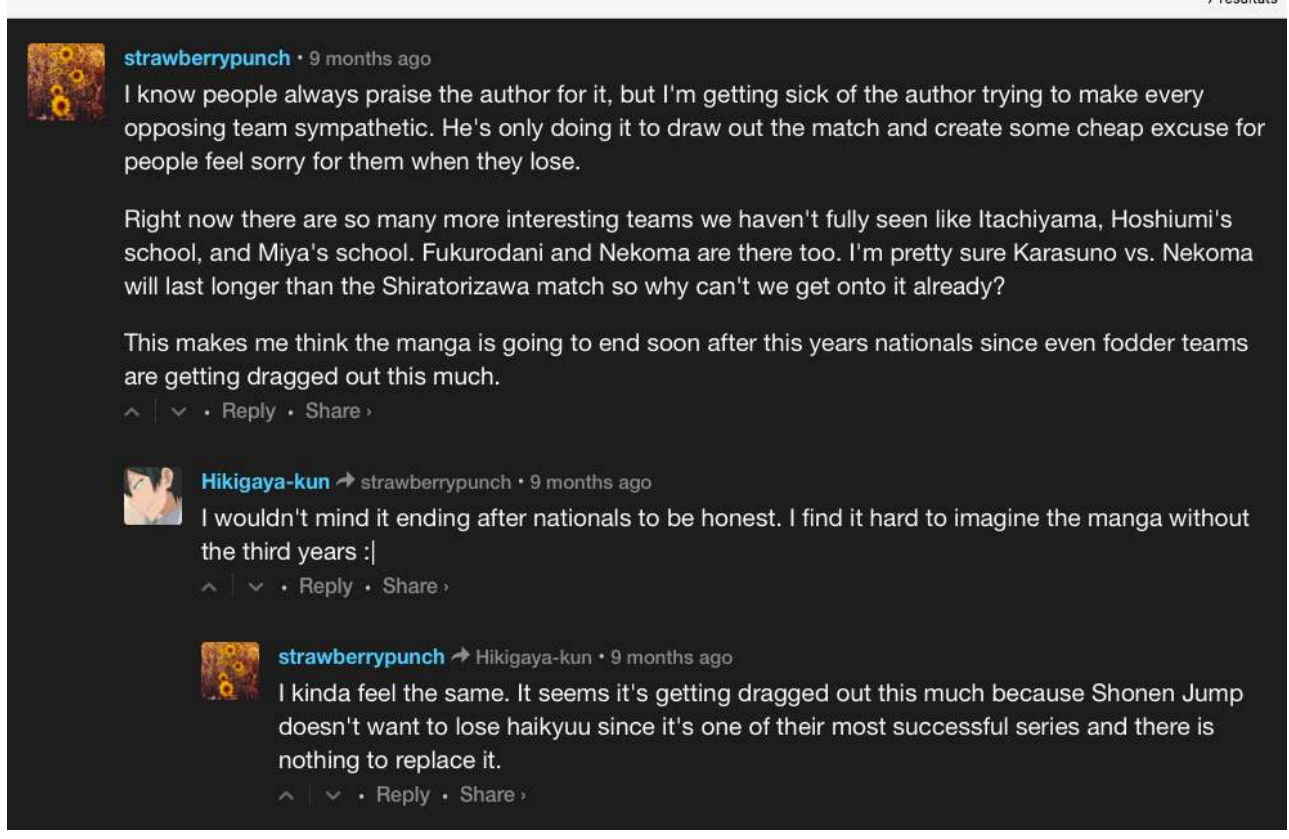

Figure 12 :Selon « Strawberrypunch », I'auteur tend à trop délayer l'action mais il y serait obligé car il s'agit d'une série populaire au sein du magazine Shonen Jump et qu'il faut la faire durer.

Comme beaucoup de séries sont ensuite adaptées en anime, les lecteurs comparent la progression en bande dessinée à celle du récit audiovisuel. Dans le cas de Dragon Ball Super, les remarques soulignent les différences de supports afin de marquer la préférence pour l'une ou l'autre version (fig. 13). Un savoir précis sur le contexte de production peut donc être collecté à travers les commentaires de ce public. L'échange sert ainsi à construire la réception en orientant l'interprétation du groupe, l'intervention savante au sein de la discussion étant une forme de distinction symbolique. 
Reader $\# 42 \rightarrow$ Chris $\cdot 2$ days ago

I for one think the manga's explanations and flow is much smoother.

For this arc alone so far, seeing all the GoDs' angels, universes, and brief interactions is far

more enlightening. Including their history with Beerus.

Toppo and Goku aren't moronic. Instead of "Oh you must be a villain because we think Zeno's destroying universes because of you" and Goku stupidly plays along "Oh I'm a villain huh?" Now everyone knows that Goku actually gave them a chance at survival and don't treat him like a dick. In the anime, they all knew and got clarified but they STILL keep that same attitude for no reason.

Much cooler to see Goku progress through all his forms like that instead of revealing SSB against a giant, filler, bs wolf side character. etc.

It's just facts

$1 \wedge \checkmark$. Reply . Share

Figure 13 : Un utilisateur expliquant pourquoi il préfère Dragon Ball Super en manga (plus grande cohérence des personnages, présentation des événements plus claire).

Il est à noter que ces interventions critiques sont surtout le fait de lecteurs presque «monomaniaques ", intervenant essentiellement au sujet d'une série ${ }^{35}$, les utilisateurs qui suivent et commentent plusieurs mangas étant moins ouvertement critiques. La passion pour un seul objet pousserait sans doute les individus à se spécialiser et à se distinguer des autres commentateurs en proposant des informations plus précises sur le monde de la production ou sur les ressorts narratifs stéréotypés d'un genre. C'est notamment en utilisant ces informations qu'ils en viennent à critiquer les auteurs, leur reprochant d'être trop conventionnels ou d'obéir aux impératifs commerciaux du magazine de publication.

Bien sûr, ce type de commentaires n'est pas majoritaire et ils ne sont pas les plus mis en valeur par le système de recommandation de Disqus. Comme l'ont souligné les nombreuses études sur les groupes de discussion, forums et blogs, l'approche distanciée n'est pas la plus fréquente et témoigne souvent d'une plus grande participation au sein de la communautés6. L'exégèse de l'œuvre permet ainsi de mettre en valeur certains individus dans une stratégie de distinction, ceux-ci affichant leur plaisir avec plus de détachement. Ils réinvestissent les critères axiologiques des productions culturelles légitimées par les institutions dans un champ populaire et médiatique en critiquant notamment ce qu'ils perçoivent comme un prolongement artificiel de l'intrigue afin de faire acheter plus de magazines (fig. 12).

\section{Savoir occidental ?}

Si les commentaires permettent de montrer comment le public construit à la fois un savoir bien lire, un univers diégétique homogène et un ensemble de connaissances sur le monde de l'édition, force est de constater qu'ils ne permettent en rien de montrer la distance culturelle entre l'œuvre et le public anglophone, comme si le manga était bien, comme le soutient Iwabuchi ${ }^{37}$, un produit odorless. Cette absence de questionnement pose problème dans le sens où tout se passe comme s'il n'y avait pas d'hétérogénéité 
culturelle entre cette communauté anglophone et le public japonais auquel les œuvres sont initialement destinées ${ }^{38}$.

Pour bien des auteurs japonais, la diffusion globale est surprenante car ils s'attendent à une incompréhension de la part des récepteurs étrangers n'ayant pas les codes pour décrypter une œuvre de culture japonaise. Pour la diffusion internationale des films Ghibli ou de Pokémon, une atténuation des éléments japonais a été opérée par les diffuseurs américains. Il est probable que le travail de traduction des mangas par les amateurs, même s'il peut contenir de nombreuses notes culturelles ${ }^{39}$, tende à effacer l'altérité de l'œuvre originale, et ce d'autant plus que ce travail des amateurs n'est pas dénué d'erreurs. Travaillant dans une perspective contrastive, la linguiste Catherine Kerbrat-Orecchioni soulignait que « chaque langue découpe et catégorise la réalité $»^{40}-$ autrement dit, l'usage de l'anglais pour traduire une œuvre étrangère et comme langue véhiculaire pour un public aux origines linguistiques diverses tend à neutraliser les différences potentielles. L'utilisation de l'anglais comme lingua franca au sein d'un groupe international effacerait les particularismes culturels de ses membres. Les connaissances produites au cours des discussions sont donc des savoirs anglais sur une œuvre japonaise, des formes d'appropriation et de resémantisation des éléments de la fiction.

Une deuxième hypothèse pouvant expliquer l'absence de friction culturelle vient du dispositif technique et du cadre choisi pour l'étude. Dans leur étude sur la réception de Dallas, Tamar et Katz montraient que les publics n'étaient pas sensibles aux mêmes éléments selon leur communautés ethniques ou leur nation d'appartenance. Les entretiens faisaient suite au visionnage de plusieurs épisodes et les publics constitués par les chercheurs discutaient dans leur langage d'origine. Dans le cas de Disqus et Kissmanga, tous les membres du public dialoguent en anglais même si les origines ethniques et les nationalités diffèrent. Ce mélange et l'usage d'une langue «véhiculaire » commune tend à renforcer une interprétation neutre, alors que dans l'étude sur Dallas les publics ne communiquaient pas entre eux. La mixité ou l'homogénéité culturelle semble ainsi jouer un rôle important dans l'expression en ligne. La formation du public autour d'un goût commun permet en quelque sorte de neutraliser la variable culturelle dans les publics dans le sens où tous les commentateurs anglophones de Kissmanga sont face à un objet culturel étranger qu'ils tentent d'interpréter au sein d'un groupe transnational. Ainsi dans les commentaires des chapitres 239 et 240, plusieurs utilisateurs interviennent en précisant qu'ils ne sont pas anglophones (fig. 14) ou qu'ils s'expriment par rapport à ce qu'ils connaissent de leur pays (fig. 2) ce qui permet de désamorcer les conflits potentiels, de confronter les points de vue et partager les expériences.

Prim Liwayway $\rightarrow$ strawberrypunch $\cdot 9$ months ago

I know they are playing in a stadium right now. What I meant was how big is the gym where the opponent practices if that player could practice a serve like that? How high is the ceiling at their own gym? Or maybe he just practices outside.

Sorry if my comment was confusing. English is not my first language. - Reply - Share

Figure 14 : Commentaire d'un utilisateur non anglophone qui souligne sa non maîtrise de la langue véhiculaire. 
31 Une dernière hypothèse est liée au phénomène d'acculturation, d'accoutumance progressive à une culture différente par le biais de la fréquentation régulière des fictions étrangères. Ce point a été souligné par une étude sur la proximité culturelle entre un public allemand et des récits audiovisuels américains.

Long running TV series in particular, might play a role in the acquisition of intercultural capital and the decrease of perceived cultural distance, as their narratives are based on an everyday culture full of cultural cues, symbols, and shared meanings. Additionally, identification processes lower the perceived distance between one's own and another culture. They allow for a casual, implicit learning about the mundane aspects of foreign cultures ${ }^{41}$.

Si dans les années 1990, un manga pouvait susciter de vives réactions de rejet de la part d'un public non préparé à cette forme de divertissement, aujourd'hui ce format de bande dessinée est populaire dans de nombreux pays non asiatiques. S'il a pu y avoir des frictions liées à des problèmes interculturels, celles-ci se sont sans doute amoindries à mesure que la lecture de manga devenait une pratique de loisir courante. En outre, le public de Kissmanga ne se compose pas de consommateurs ordinaires. Il s'agit d'un groupe d'amateurs ayant déjà une connaissance spécialisée dans la culture populaire japonaise puisqu'ils suivent régulièrement leurs séries sur une plateforme non légale au lieu d'attendre la publication officielle dans leur territoire. Cette familiarité avec certains codes culturels japonais transmis à travers les fictions leur permet sans doute de dialoguer et communier malgré une absence d'interconnaissance.

\section{Conclusion}

L'analyse des commentaires de lecteurs sur le site Kissmanga permet ainsi de voir que le public anglophone construit et partage une série de savoirs sur le manga. Outre un « savoir bien lire » se marquant par la mise en scène de l'émotion ressentie à la lecture, ils échangent des savoirs sur le monde diégétique et le monde de production: des connaissances et interprétations sur les personnages, la construction d'une cohérence narrative par rapport à un genre ou d'autres versions transmédiatiques du récit, des analyses sur l'impact du monde réel (que ce soit les règles du volley ou les conditions de production) sur la création de la diégèse. L'ensemble se produit sur un mode délibératif qui associe une écriture créative où jeux de mots et jeux de rôle alternent pour le plaisir de la conversation. Les informations sont ensuite reprises et consolidées dans des formes d'écriture plus formatées : wiki, articles de blog ou de sites spécialisés. Ces commentaires participent au plaisir de la lecture en ligne, celle-ci étant motivée à la fois par l'envie de connaître la suite du récit mais aussi par la possibilité d'en parler avec d'autres afin de s'approprier la fiction par le biais de la conversation.

Mais ce lieu virtuel de sociabilité suscite également plusieurs questions. Outre la difficulté à étudier de façon sociologique ce public transnational en raison de l'anonymat, il faut souligner l'importance de l'interface dans la formation de cette communauté. Les conversations auraient-elles été différentes si Disqus mettait en avant les propos de personnes géographiquement (et potentiellement culturellement) proches au lieu d'afficher les commentaires de manière chronologique ou selon le degré de "popularité » (nombre de +1 attribué par les internautes) ? La relative simplicité et l'immédiateté des dialogues transnationaux en ligne tendent à effacer la complexité du dispositif technique et son rôle dans la constitution même des discussions. Etant donné l'importance économique de celles-ci pour la société gérant 
Disqus et les sites qui utilisent l'outil pour fidéliser les internautes, il serait nécessaire d'étudier comment la plateforme monétise ces commentaires et les favorisent. L'apparente transparence et immédiateté des échanges cache en réalité une série de médiations techniques liées à la plateforme de communication qui influencent les conversations et la réception d'une œuvre.

Enfin, il me semble que l'on peut s'interroger sur l'hégémonie de l'anglais comme langue véhiculaire entre des publics occidentaux et des productions culturelles japonaises dans ces réseaux de diffusion non légaux. Sa pratique est inégale selon les pays et les catégories socio-professionnelles, ce qui entraîne une sélection des utilisateurs potentiels de ces circuits.

\section{NOTES}

1. Anne-Sophie Béliard, «Jeux croisés entre critique amateur et critique professionnelle dans les blogs de séries télévisées ", Réseaux, 32, 2014, pp. 93-123 ; Dominique Legallois, Céline Poudat, «Comment parler des livres que l'on a lus? Discours et axiologie des avis des internautes", Semen, 26, 2008.

2. Bien sûr, il faut se garder de croire en une transparence totale du médium numérique comme le souligne Matt Hills : "This represents what I would term a serialisation of the fan audience itself. The term is intended to indicate that the online X-Files audience cannot merely offer a 'window' on the programm's offline, socially atomised fandom; it must, instead, perform its fan audiencehood, knowing that other fans will act as a readership for speculations, observations and commentaries ». Matt Hills, Fan Cultures, London, Routledge, 2002, p. 139.

3. Henry Jenkins, “'Do You Enjoy Making the Rest of Us Feel Stupid?': alt. tv. twinpeaks, the Trickster Author, and Viewer Mastery ", in Henry Jenkins, Fans, Bloggers, and Gamers. Exploring Participatory Culture, New York, New York University Press, 2006, pp. 115-134.

4. Matt Hills, op. cit.

5. Laurence Allard, «Cinéphiles, à vos claviers! Réception, public et cinéma », Réseaux, 99, 2000, pp. 131-168.

6. Valérie Beaudouin, Dominique Pasquier, «Organisation et hiérarchisation des mondes de la critique amateur cinéphile », Réseaux, 183, 2014, pp. 125-159.

7. L'étude porte sur les commentaires des lecteurs de Haikyû!! et Dragon Ball Super sur la période de juin à novembre 2017 sur le site Kissmanga.

8. Les études sur le fonctionnement de Wikipédia sont nombreuses. On peut notamment citer Julien Levrel, «Wikipédia, un dispositif médiatique de publics participants », Réseaux, 138, 2006, pp. 185-218. Et le livre plus récent de Lionel Barbe et alii, Wikipédia, objet scientifique non identifié, Nanterre, Presses universitaires de Paris Nanterre, 2015.

9. Le sociologue s'intéressait aux conditions de la formation et de l'expression des goûts dans l'espace public en postulant que la « réception n'a d'existence sociale que sous forme de discours ». Dominique Boullier, La Télévision telle qu'on la parle. Trois études ethnométhodologiques, Paris, L'Harmattan, 2003, p. 14.

10. Matt Hills, op. cit., p. 140.

11. Tamar Liebes, Elihu Katz, The Export of Meaning: Cross-Cultural Readings of Dallas, Cambridge, Polity Press, 1994. 
12. Sur ce site, 12220 séries de mangas étaient disponibles en traductions anglaises non légales en novembre 2017. Dans le cadre de cette étude, nous nous intéresserons plus particulièrement aux lecteurs de la série Haikyû!!, actuellement publiée dans le magazine Shônen Jump.

13. Contrairement à Facebook et son plugin «Comments" implémenté sur certains sites non légaux, Disqus accepte les pseudonymes et propose donc une plateforme de conversation publique et anonyme. Ce détail explique en partie la différence de participation des utilisateurs : l'anonymat favorise les interactions.

14. Sur les pratiques transplateformes voir Mélanie Millette, "Pratiques transplateformes et convergence dans les usages des médias sociaux ", Communication et organisation, 43, 2013, pp. 47-58.

15. Ce travail de suivi des discours sur Twitter à partir d'un hashtag a été réalisé par Laurence Allard au sujet de la série Black Mirror. Laurence Allard, « Voir et dire la fiction. Vers un double agir communicationnel, \#blackmirror \#LT », in Châteauvert et Delavaud, op. cit., pp. 501-514.

16. En moyenne, après chaque chapitre hebdomadaire d'un best-seller comme One Piece, il y a 550 commentaires (en sachant qu'il y a plus de 800 chapitres et que la série est inachevée). Haikyû!! ne recueille que 90 commentaires hebdomadaires (plus de 300 chapitres et toujours en cours de publication). À titre de comparaison les séries à périodicité mensuelle comme Dragon Ball Super ou L'Attaque des titans reçoivent en moyenne entre 150 et 160 commentaires.

17. La série sur le volleyball Haikyū!! (ハイキュー!!) est orthographiée Haikyu!! dans la version officielle anglaise et Haikyû!! en français. Mais elle est également orthographiée Haikyuu!! dans une majorité des sites non légaux. Dans la suite du texte, le titre est uniformisé avec la graphie française.

18. Certains utilisateurs consultent les vidéos dans leur établissement scolaire, d'autres mentionnent les interrogations des parents face à leur réaction durant le visionnage, ce qui donne des indications sur l'âge des utilisateurs. Le profil Disqus permet également de savoir si l'utilisateur commente beaucoup, s'il est suivi par d'autres, participe régulièrement, etc. Ce profil regroupe aussi en un seul lieu tous les commentaires de l'utilisateur, ce qui nous permet de voir à quel point ses textes changent en fonction du temps passé à discuter, le nombre de séries lues et commentées.

19. Une fois connectée à Disqus les internautes peuvent participer aux conversations informelles ayant cours après chaque chapitre. Le dispositif technique étant proche de celui d'un réseau social, les utilisateurs pouvant faire remonter les commentaires qu'ils apprécient le plus en ajoutant un +1 , y répondre par un nouveau message. De cette manière, de nouvelles hiérarchies se constituent en fonction de l'intensité de l'activité de contribution, la qualité des commentaires et de l'engagement dans la sociabilité de réseau.

20. Benedict Anderson, Imagined communities: Reflections on the origin and spread of nationalism, London, Verso Books, 2006.

21. En sachant que la majorité du public ne laisse pas de commentaires, ce chiffre témoigne à la fois du succès de la série et de cette plateforme de diffusion parallèle.

22. Pour l'exemple d'utilisation de blogs dans l'enseignement, voir Pierre Moinard, « Commenter des textes littéraires en collaboration sur des forums et des blogs dans le secondaire ", Le Français aujourd'hui, $\mathrm{n}^{\circ}$ 196, 2017, pp. 71-80.

23. Paul Lazarsfeld et Elihu Katz, Personal Influence: The Part Played by People in the Flow of Mass Communications, New York, Routledge, 2017.

24. Cité par Allard, op. cit.

25. Lorsqu'un problème technique a effacé les dialogues sur les premiers chapitres de Haikyû!!, les utilisateurs se sont mis à remettre des commentaires afin de rebâtir l'espace de convivialité.

26. Voir Claus Dieter Rath, «Live/life: Television as a generator of events in everyday life ", dans Philip Drummond \& Richard Patterson (eds.), Television and its Audience: International Research 
Perspectives; a Selection of Papers from the Second International Television Studies Conference London, London, British Film Institute, 1988, p. 32-37.

27. Gabriel Tarde, L'Opinion et la foule, Paris, Alcan, 1901.

28. Ainsi les fans du personnage d'Oikawa interviennent régulièrement pour rappeler son antagonisme avec Kageyama afin de montrer en quoi ce premier opposant a été utile dans les progrès de celui-ci ou en quoi il est bien plus doué ou sympathique que les opposants actuels. Ces interventions régulières des fans font vivre le personnage bien qu'il n'apparaisse plus dans le manga depuis une trentaine de chapitres.

29. Voir http://haikyuu.wikia.com/wiki/Tōru_Oikawa/Relationships

30. Richard Saint-Gelais, Fictions transfuges. La transfictionnalité et ses enjeux, Paris, Éditions du Seuil, 2011, p. 386.

31. Cette pratique du Role Play n'est pas spécifique à Haikyû!! ou à l'interface conversationnelle Disqus. Mais dans cet espace, les conversations et les interventions RP ne sont pas coordonnées entre les utilisateurs, contrairement à ce qui peut se passer sur des plateformes comme Tumblr. Voir Ann McClellan, "A case of identity: Role playing, social media and BBC Sherlock ", Journal of Fandom Studies 1, 2, 2013, p. 139-157 ; Jen Scott Curwood, " “The Hunger Games": Literature, Literacy, and Online Affinity Spaces ", Language Arts, 90, 6, 2013, p. 417-427.

32. Voir par exemple celle de «JustAnotherReviewer» https://www.youtube.com/watch? $\mathrm{v}=$ HRNrUezHOT8 et celle de «Nerdigans Inc.» https://www.youtube.com/watch?v=X8q5$\mathrm{MvJC} 8 \mathrm{w}$

33. Voir celle de Reddit https://www.reddit.com/r/haikyuu/comments/5qchmn/ haikyuu_chapter_239_discussion/ et celle de Myanimelist https://myanimelist.net/forum/? topicid $=1587630$

34. Voir http://haikyuu.wikia.com/wiki/Baptism

35. L'utilisateur Strawberrypunch n'intervient que dans les commentaires de Haikyû!!.

36. Voir Beaudouin et Pasquier, op. cit.

37. Koichi Iwabuchi, Recentering Globalization. Popular Culture and Japanese Transnationalism, Durham, Duke University Press Books, 2002.

38. Il y a bien sûr des débats entre commentateurs sur l'importance de telle ou telle séquence ou personnage. Mais il ne semble pas y avoir de frictions liées à des problèmes interculturels.

39. Les notes "culturelles" sont des notes de bas de page où les traducteurs explicitent des éléments liés à la vie quotidienne japonaise, des éléments historiques ou d'autres aspects qui peuvent paraître inédits. Cette pratique de la note est spécifique des traductions de fans. Voir à ce sujet, Matteo Fabretti, "The Use of Translation Notes in Manga Scanlation », TransculturAl, vol. 8.2, 2016, p. 86-104.

40. Catherine Kerbrat-Orecchioni, Les Actes de langage dans le discours, Théorie et fonctionnement, Armand Colin, 2012, p. 169.

41. Daniela Schlütz et al., « Transnational media representations and cultural convergence - An empirical study of cultural deterritorialization", Communications, 42, 2017, p. 47-66: "Plus précisément, les séries télévisées au long cours contribuent sans doute à l'acquisition du capital interculturel et à la diminution de la distance culturelle perçue, car elles incorporent des représentations de la vie quotidienne qui regorge de signaux culturels, de symboles et de significations partagées. De plus, les processus d'identification réduisent la distance perçue entre la culture du récepteur et celle des personnages. Ils facilitent un apprentissage implicite du quotidien de civilisations étrangères » (ma traduction). 


\section{ABSTRACTS}

A travers l'analyse des commentaires de lecteurs sur un site de traductions non légales de mangas, nous visons à montrer de quelle manière la conversation fait partie intégrante du plaisir de la fiction. Partage des émotions, diffusion d'informations, débats autour d'interprétations, tout ceci contribue à la fois à constituer une communauté mais aussi à faire émerger plusieurs formes de savoirs qui seront ensuite déployés sur d'autres plateformes.

Ces commentaires témoignent des compétences génériques et des connaissances sur les processus de production. Les échanges servent ainsi à construire la réception au sein du groupe, tout en apportant à certains de ces exégètes amateurs une forme de distinction symbolique.

\section{INDEX}

Mots-clés: piratage, manga, amateur, savoir, public

\section{AUTHOR}

\section{BOUNTHAVY SUVILAY}

Doctorante sous la direction de Marie-Ève Thérenty (RIRRA21, Montpellier 3) et de Matthieu Letourneux (PHisTeM-CSLF, Paris-Ouest), Bounthavy SUVILAY étudie la circulation transnationale de récits transmédiatiques. Elle s'intéresse notamment aux adaptations de la licence Dragon Ball sur tous les supports (manga, anime, jeux vidéo, jouets) et leur (re)création et/ ou resémantisation par les éditeurs et les amateurs en France. 